\title{
CP-174 Healthcare professionals' knowledge of intravenous fluid treatment
}

\author{
S Thevelin, G Miller, E Guthrie, J Portlock
}

\begin{abstract}
Background Intravenous (IV) fluid treatment is a routine but essential part of care for a significant proportion of hospitalised patients. However one in five hospitalised patients suffer from fluid-related complications with associated morbidity and mortality. In 2013, a NICE clinical guideline was published with the aim of improving IV fluid management. A lack of knowledge was identified as a key factor contributing to poor fluid management.

Purpose To assess the knowledge among nurses, junior doctors and pharmacists of IV fluid treatment in adults. Knowledge was assessed of the daily fluid and electrolyte requirements and composition of commonly-used IV fluids.

Material and methods The study was conducted as a self-administered questionnaire survey. An anonymous questionnaire in multiple-choice format was designed based on the NICE guideline and existing questionnaires. Data collection was carried out over a four-week period in June 2014. Responses were marked as correct, incorrect or unsure.
\end{abstract}

Results 193 healthcare professionals including 96 nurses, 45 junior doctors and 52 pharmacists completed the questionnaire. $17 \%, 33 \%$ and $54 \%$ of nurses, junior doctors and pharmacists correctly identified the daily water requirements. Respectively $8 \%, 33 \%$ and $68 \%$ of nurses, junior doctors and pharmacists accurately estimated the daily sodium requirements. Only $16 \%$ of nurses, whereas $71 \%$ of junior doctors and $94 \%$ of pharmacists correctly identified the daily potassium requirements. The knowledge of the composition of commonly used IV fluids was poor, which is of particular concern as this guides fluid prescription. The inadequate knowledge of junior doctors confirms the findings of previously published studies, whereas to current knowledge no studies have been published with regards to nurses and pharmacists.

Conclusion Despite the development of a new guideline, knowledge of IV fluid treatment remains poor. Measures are needed to increase awareness and reduce the high number of fluid-related complications.

References and/or Acknowledgements No conflict of interest. 